\title{
Use of Energy Storage for Belgian Power Network
}

\author{
Mohammad Moradzadeh*, Brecht Zwaenepoel ${ }^{\dagger}$, René Boel ${ }^{\ddagger}$ and Lieven Vandevelde ${ }^{\S}$ \\ * Electrical Energy Laboratory, Dep. of Electrical Energy, Systems and Automation, \\ Ghent University, 9000 Ghent, Belgium, mohammad.moradzadeh@ugent.be \\ $\dagger$ Energy knowledge platform Power-Link \& Electrical Energy Laboratory, \\ Dept. of Electrical Energy, Systems and Automation, Ghent University, 8400 Oostende, Belgium \\ $\ddagger$ SYSTeMS Research Group, Dep. of Electrical Energy, Systems and Automation, \\ Ghent University, 9000 Ghent, Belgium \\ $\S$ Electrical Energy Laboratory, Dept. of Electrical Energy, Systems and Automation \& \\ Energy knowledge platform Power-Link, Ghent University, 9000 Ghent, Belgium
}

\begin{abstract}
The intermittent and fluctuating nature of output power of renewable energy sources (RES) can lead to serious security concerns such as congestion in the transmission system. Incorporating storage devices with RES is technically an effective means to mitigate this concern by allowing fluctuating RES to be as stable as traditional power plants. Storage is an even more consumer-friendly solution with greater control over electricity consumption time and price compared to e.g. demand-side management which offers limited control over the price for end users. It can also contribute to lower the electricity price, peak-shave the demand, defer upgrade investments, provide ride-through solution for (momentary) faults, contribute to voltage/frequency control, increase the share of green energy and thus reduce overall greenhouse gas emissions, improve power quality by reducing harmonic distortions and voltage sags/surges, facilitate utilization of more efficient off-peak generation units, etc. This paper assesses the technical applicability and economical viability of different storage devices to the Belgian power network, focusing on the structure of the Belgian electricity market. A high-level overview of widely-used storage technologies, their benefits and shortcomings are also provided.
\end{abstract}

\section{INTRODUCTION}

Energy storage is becoming an essential element in today's electrical power systems. Electricity is a man-made (and nonnatural) commodity that cannot be stored on a large scale in an economically viable sense. Efficient and (at the same time) economic transmission of electricity over long distances is also a concern. This means that the electricity production must be fine-tuned to consumption levels on a short-term basis (often every 15 minutes) as so to guarantee that the supply and demand are always balanced. This task is indeed the greatest responsibility of the Transmission Systems Operator (TSO), an organization which is independent of other commercial players in the competitive electricity market such as producers/suppliers, traders and retailers. Today, with the increasing penetration of Renewable Energy Sources (RES), the variable and intermittent nature of output power of RES arises serious reliability concerns regarding their seamless supply. The output power of wind turbines and solar panels varies considerably due to climate conditions as the wind does not blow and the sun does not shine all the time. This (unpredictable) uncontrollability in output power of most of RES can be somehow mitigated by introducing a storage component to RES in order to decouple the instantaneous generation from the consumption. A Hybrid Renewable Energy System (HRES) is defined as a system consisting of single/multiple RES along with a (or a combination of several) storage element(s) [1]. Some examples are commercialized stand-alone solar-battery configuration for street lighting, or a wind-solar-fuel cellhydrogen storage configuration for electrifying a remote rural area or an island. In the context of microgrids, storage can also be used as a possible countermeasure for transmission congestion management, where there is too much excessive power produced by e.g. wind and solar units beyond the maximum capacity of lines to transport that much power. In this regard, the storage can be effectively used to store otherwise curtailed surplus green energy during the congested periods alleviating the congestion-induced wind/solar curtailment [2]. Note that generation curtailment may be necessary due to other factors as well such as low demand, underestimated wind forecast and/or network security reasons. The stored energy can be economically sold to a spot market when technically (transmission) network capacity is available for price-arbitrage purposes. It is also noteworthy to mention that (wind) curtailment at first sight might seem as an ignorant waste of green energy which is indeed a lost revenue for the turbine owner. However, considering that congestion-induced curtailment does only become necessary in a relatively short time span of the year for a limited number of wind units, curtailment can provide a suitable tool to allow connecting additional RES to the grid during a lot longer non-congested periods, ultimately increasing the capacity share of green energy.

The techno-economic assessment of different available storage technologies is of utmost concern when deciding upon their use. This paper aims at outlining the different storage technologies and their characteristics, and discusses their potential application to the Belgian power network.

The remainder of this paper is orginised as follows. In section II, first the electricity market operation is clarified, and then the role of storage in mitigating risks arising from integration of RES into electricity market is discussed. Section III provides an overview of widely-used energy storage technologies, their benefits and shortcomings. The potential applicability of different storage elements to the Belgian power network is discussed in section IV. Finally, the conclusions are drawn in section V.

\section{StORAGE MARKET INTEGRATION}

Market integration of RES heavily depends on the predictability of these sources as electricity cannot be stored directly. Energy trading relies on forward markets to buy and sell energy from days, weeks, months or even years before actual delivery. Hence, increasing predictability will reduce the costs of the imbalance fines. 


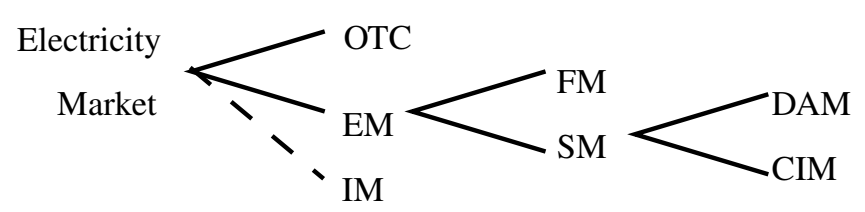

Fig. 1. Energy trading markets

\section{A. Electricity market structure}

Suppliers and retailers (or large consumers) have different possibilities to trade electricity, as shown in Fig. 1. First of all, they have the possibility to trade Over The Counter (OTC) where the contract is made directly between the seller and the buyer. A major part of the total traded volume is sold by bilateral contracts. Especially long-term base load or predictable load is traded directly between supplier and retailer/consumer. No intermediate parties are involved.

The second option is to trade energy on exchange market (EM) which can further be classified into two largely distinctive markets. The first one is the futures market (FM). Futures are contracts to deliver a certain quantity of electricity on a date in the future. This is an easy way of trading large quantities of base capacity, as these contracts are often concluded for months or years at a time. The second exchange market is the spot market (SM). On this market, energy is traded close to the delivery time. The spot market has two separate parts: the Day Ahead Market (DAM) [3] and the Continuous Intraday Market (CIM) [4]. Of the two, DAM is most widely used due to technical constraints of the CIM. As the name suggests, on the DAM, the energy is traded the day before delivery. Sellers and buyers submit their supply and demand curves. Once a day, these are matched via an auction process and a price for each trading hour of the following day is determined. This ensures an anonymous and transparent price fixing process. In contrast, the CIM provides the parties a platform to trade energy very close to the delivery time, up to a few minutes before actual delivery. Since for every physical transfer on the transmission grid acknowledgment of the grid operator is additionally needed, this is a limited market. The CIM is only used to settle large unbalance positions after gate closure on the DAM (e.g. production unit breakdown). As a result, often only a few hours a day energy is traded in this market. In Belgium, recently the transmission system operator (Elia) made also the Imbalance Market (IM) available. The possibility to trade on this market is also limited as it depends on the system state (net regulation volume).

In Europe, several markets are connected. Since 17 January 2011, five exchange markets are effectively coupled: APXENDEX (The Netherlands), EPEX Spot FR (France), EPEX Spot DE (Germany), EMCC (Europe) and Belpex (Belgium). As a result, these markets share a common price as long as cross border capacity is available [5]. When the physical transfer capacity between the market areas is constrained, different price zones are formed.

\section{$B$. The role of storage in RES integration}

RES present new challenges to the existing energy markets. Traditionally, production and consumption could be predicted accurately [6]. This made it possible to plan the production

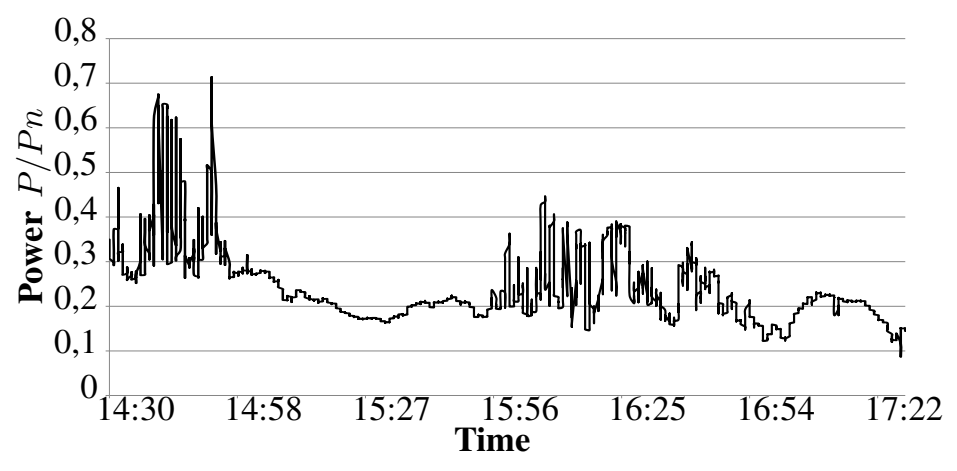

Fig. 2. Variability of RES (Solar)

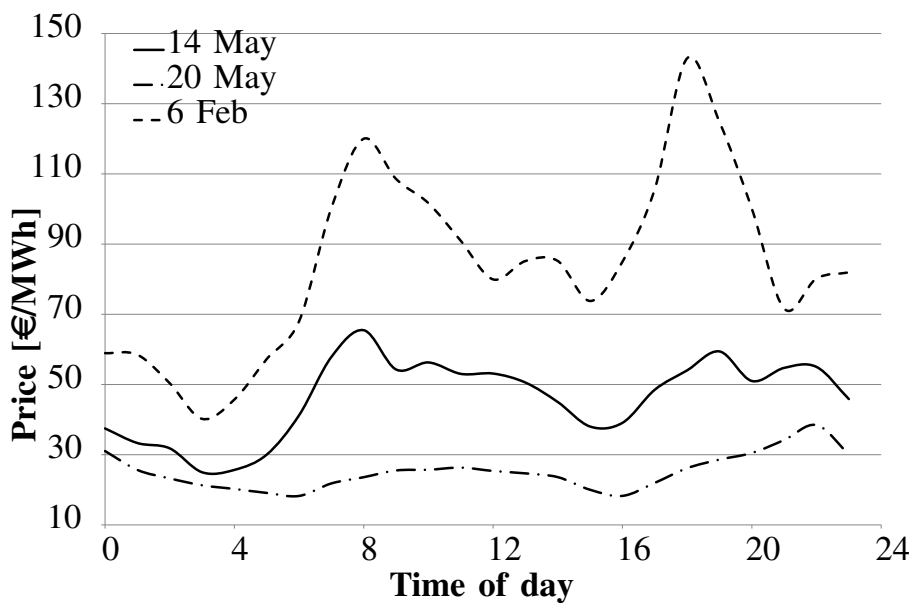

Fig. 3. Evolution of electricity price on three different days in 2012 (Belpex)

schedules of power plants days and even weeks or months beforehand. However, as RES are often dependent on weather conditions which are more difficult to accurately predict, the real and predicted power flows differ more. This variability and more specifically the unpredictability makes efficient market integration of RES to the existing markets more difficult. Fig. 2 illustrates the intermittency of output power of a solar panel over several hours on 10 October 2011 in Oostende in Belgium. Additionally, the markets are designed for large quantities of energy, rounded to $0.1 \mathrm{MW}$ and time slots from $15 \mathrm{~min}$. to $1 \mathrm{~h}$. Storage can alleviate the unpredictability of RES and provide more certainty towards the markets.

Two distinct optimization goals for the storage device can be defined: short-term optimization to dampen power fluctuations and avoid penalties for imblanace prediction errors, and long-term optimization to make maximal profit. Short-term optimization can be used to mitigate the variable output of e.g. solar panels or wind turbines. In this way, storage can alleviate the difference between the nominated and effectively traded volume, and hence can avoid the balancing cost [7]. As Balance Responsible Parties (BRPs) need to balance their production/consumption and import/export -sayevery fifteen minutes, only relative small amounts of storage are needed to level the actual and the predicted energy balance. In [8] a multi-objective Particle Swarm Optimization (PSO) is proposed to find the best PV-battery-H2 configurations in order to design a autonomous stand-alone HRES for a realistic case of a remote village called La Nouvelle in the French overseas island of La Réunion, in both short-term (2 years) 
and mid-term (10 years). In [2] an Linear Programming (LP) problem is solved to optimize the operation of energy storage for wind curtailment reduction and price-arbitrage, on a case study of $33 \mathrm{kV}$ distribution network in the UK.

Storage units with a larger energy content can be used to sell energy on the most profitable moments on the market. As can be seen in Fig. 3, price evolution on the stock market can differ significantly from day to day and from month to month. Storage intended for market optimization can be regarded as any storage from hours to even months. In case large price differences in one day are available (e.g. profile of 6 February 2012 in Fig. 3), storing energy produced in low price zones to sell the energy in time slots with high prices can be profitable. However, on days with a relatively flat price profile (e.g. profile of 20 May 2012 in Fig. 3), no significant financial gain should be expected from storage. In [9] a price-baced unit commitment strategy is presented to optimise total profit for a collection of Distributed Energy Resources (DER). This strategy utilises a Virtual Power Plant (VPP) to consolidate different DER units to form a single entity.

\section{OVERVIEW OF ENERGY STORAGE TECHNOLOGIES}

Electrical energy storage is accomplished by devices or physical media through converting it into another form e.g. chemical, thermal, potential or kinetic energy. There exist different types of energy storage technologies which may suit grid-scale applications or small-scale application (e.g. (large) commercial/residential dwellings). Comparison among these highly application-dependent energy storage technologies seems to be pointless, and their use should, as a simple rule of thumb, be decided based upon an in-deep economical capital/maintenance cost analysis, efficiency (taking the losses into account) and/or their maturity/infancy of technology. This section provides a high-level overview of the most widelyused storage technologies namely pumped hydro, compressed air energy storage (CAES), flywheel, battery, fuel cell (FC), hydrogen storage, thermal energy storage (TES), and (super)capacitor.

\section{A. Pumped-hydro energy storage (PHES)}

A conventional PHES unit uses the height difference of two vertically-separated natural/man-made water reservoirs. A reversible pump-turbine with motor-generator set is employed to function in both charging mode (to drive the pumps) and discharging mode (to run the turbine). Excess (off-peak lowprice) energy is used to pump the water from the lower reservoir to the upper reservoir storing electricity in the form of potential (gravitational) energy of water. During peak periods, the potential energy is released by reversing the water flow to turn the turbine in order to produce electricity. Some conventional hydro-electric power plants which only use the natural stream flow of water (no water pumping) may be also dispatched as a PHES facility by deferring the electric power output until required.

Pumped-hydro is technologically a very mature solution, being practically the most widespread energy storage system in current use in power systems accounting for more than $99 \%$ of bulk storage capacity worldwide. It is generally the most costeffective solution for large-scale energy storage being available in the order of hundreds of MW and MWh. PHES system provides a suitable solution for long-term energy storage with the discharge times ranging from several hours to a few days with efficiency of $70 \%-85 \%$. They have fast response time achieving high ramp rates, long lifetime (e.g. 50 years). The disadvantages, however, are their long/time-consuming construction time, high capital expenditures, inevitable time needed before switching between charge/discharge modes due to significant inertia of the water, and dependency on the availability of an appropriate geographical reservoir [10].

\section{B. Compressed air energy storage (CAES)}

A CAES system uses the excess (green) electricity during off-peak periods to power an electric motor functioning as air compressor. Compression of air to high pressure produces heat which is captured by a thermal energy storage unit. The compressed air is often stored in underground geological reservoirs such as salt caverns, rock mines, depleted oil and gas wells. During peak hours or high demand periods, the stored compressed air is re-heated using the excess heat which was created (and captured) during compression phase in the thermal store unit and then expanded in the turbine, in order to (in)directly generate electricity. Note that most existing CAES systems are installed in conjunction with natural gasfired power plants, where the compressed air is fed into the gas turbine to boost efficiency (indirectly generating electricity). There are three types of CAES systems, depending on the thermodynamics of the process, namely adiabatic (isentropic), diabatic and isothermal:

- Diabatic or conventional CAES dissipates reasonable amount of the heat into the environment, which is produced during compression phase. They thus need to re-heat the compressed air prior to expansion in the turbine. This is often done by using the heat produced by natural gas-fired (combustion) turbine to heat the compressed air.

- Adiabatic CAES retains the heat produced during the compression phase to re-heat the compressed air during the expansion phase in order to generate electricity. The system is insulated against heat transfer to the environment avoiding the resulting energy losses.

- Isothermal CAES tends to keep operating temperature of the air at a constant level throughout compression/expansion processes by constant heat exchange to the environment. It allows air to be compressed to high pressures at near ambient temperature.

The CAES system provides a suitable solution for large-scale long-term storage as the small-scale CAES systems are not commercially available at the present time. A notable advantage of CAES is that air, unlike e.g. electrodes and electrolytes in batteries, is naturally available at zero marginal cost. The energy density, in $\mathrm{kWh} / \mathrm{m}^{3}$, is relatively high compared to e.g. pumped hydro storage system. One of the limitations is that it often takes several minutes to switch between charge and discharge modes. Another disadvantage is that the economical viability of CAES system depends on geographical-based natural formations. 


\section{Flywheel}

A flywheel is nothing more than a rotor (flywheel) that can spin around a shaft at a very high speed. The rotor is a high-inertia cylindrical assembly made of steel or carbonfiber composite that is directly coupled to a shaft on which a motor/generator is mounted. The excess green energy, during charging mode of the flywheel, is used to accelerate the rotor to a very high speed (e.g. $50000 \mathrm{rpm}$ in some applications), with the motor/generator set acting in motor mode, and energy is stored as kinetic energy in the rotor. The amount of kinetic energy that a flywheel can store $\left(E_{\text {flywheel }} \simeq m r^{2} \omega^{2}\right)$ is proportional to flywheel's mass $m$, the square of rotor's radius $r$ and the square of angular velocity of the rotor $\omega$. During peak periods, the motor/generator set is switched into generator mode, and the initial kinetic energy of the flywheel drives the generator in order to produce electricity, causing the flywheel to discharge and thus to slow down. In order to reduce the losses associated to friction, the rotor assembly is sealed in a vacuum chamber, and is additionally levitated with an electromagnetic bearing. Flywheels have high efficiency (about $85 \%$ ), very high power/energy densities (power/energy per unit volume), fast (near immediate) response, long lifetime under constant cycling and low maintenance cost. They are very environmentally-friendly and can be applied to a wide range of applications. Considering very high specific power (power per unit mass) of flywheels, they seem to be an ideal solution when a fast charge/discharge of large quantity of energy is required, and when the overall energy capacity is not a critical issue. Flywheel technology has gained some commercial applications in automotive and rail industry for regenerative braking. Disadvantages are high capital cost, design complexity, overall system size and weight, possible destructive damages upon failure.

\section{Battery}

A (rechargeable) battery, in most basic form, consists of several electrochemical electrically-connected (in series or parallel) stacked cells that convert electricity into chemical energy and vice versa. Each cell contains electrodes (negatively charged (anode), positively charged (cathode)) wherein reduction-oxidation (redox) reactions take place, and an electrolyte. There exist many different type of batteries depending on various chemicals used as electrodes and electrolyte. Upon discharging, a chemical reaction takes place inside the cell and the released electrons travel from anode to cathode supplying the load. When recharging the battery the reverse process occurs and again electricity is stored as chemical energy in the cell. Some well-known battery types are: lead-acid $(\mathrm{Pb}$ A), nickel-cadmium (Ni-Cd), nickel metal hydrite (NiMH), lithium-ion (Li-ion), sodium-sulphur (Na-S), vanadium redox flow and metal-air. Note that energy storage devices are normally rated in terms of both their energy (expressed in $\mathrm{kW}$ or MW) and their power capacities (expressed in $\mathrm{kWh}$ or MWh) as it is equally important to know how long a battery can supply a given capacity of energy. Unfortunately, the energy and power capacities cannot be scaled up/down independently for most battery types as they are strongly correlated. Several important technical specifications are often used to characterize and compare battery operating conditions such as (roundtrip) efficiency, cycle life (lifetime/lifespan), depth of discharge, self-discharge, energy/power density, specific energy/power.
Battery storage is a relatively nascent technology. They have high cost, relatively small capacity, high maintenance and limited lifetime. Detailed techno-economic comparison of different battery types can be found in [14], [15], and are not discussed here.

In conventional batteries electrolyte is in direct contact with the electrodes (of the electrochemical cell/stack) as they are both contained in the same container. Flow battery is a special class of batteries in which the electrolytes (two different types) are separately stored in external storage tanks outside of the cell (electrodes and membrane separator) itself. To charge/discharge flow battery, the electrolytes should flow through the electrochemical cell by pumps. Thanks to this physical structure, the energy and power densities of flow batteries can be scaled up/down independently. Because the energy density is determined by the amount of separately stored electrolytes (size of the storage tanks), while the power density is independently determined by the rate at which chemical redox reactions occur at the electrodes inside the cell. This feature is very important for design optimization of energy and power densities for every specific application. Vanadium redox, regenerative fuel cell and zinc-bromin are three different types of flow batteries. They provide largescale long-term storage capacity, and have no self-discharge. Disadvantages are their low efficiencies (due to consumed energy to run the electrolyte pumps) and design complexities.

\section{E. Fuel cell $(F C)$}

A fuel cell is an electrochemical energy conversion device that converts the chemical energy of a fuel, often hydrogen, into DC electricity (and water and heat as by-products) through chemical reaction with e.g. oxygen of the air. An FC generally consists of two electrodes (anode and cathode), an electrolyte and a catalyst. The chemical reactions take place at the electrodes, at the presence of catalyst to speed up the reactions, and an electrolyte which only allows the passage of appropriate ions between electrodes. In practice, single cells are often assembled together in series to form a stack. FC technologies in principle differ in type of electrolyte they use, and generally operate at different temperatures. A possible general classification for different FC technologies is given below:

- low temperature FC: alkaline FC (AFC), phosphoric acid FC (PAFC) and proton exchange membrane FC (PEM or PEMFC)

- $\quad$ high temperature FC: (molten) carbonate FC (CFC or MCFC) and solid oxide FC (SOFC)

The operating temperature determines the applicability of the FC technology to a particular application, as well as whether or not a particular fuel can be utilized. For example typical operating temperature of a SOFC (about $1000 \mathrm{C}^{\circ}$ ) limits its safe use for homes and cars. Furthermore, low temperature FCs operate with hydrogen, while high temperature FCs can also be operated with different hydrocarbons e.g. natural gas or alcohols e.g. methanol. Fuel cells cannot generally store energy unless combined with electrolyzers and external storage systems.

FCs are clean with zero (in case of using pure hydrogen as fuel) or near-zero emissions, can achieve high efficiencies at 
any scale, and can be located almost anywhere. The efficiency can be even further improved if the waste heat of the FCs can be harnessed. FCs output scalable high power density such that doubling the operating time needs only doubling the amount of input fuel and not doubling the capacity of FC itself. They generally require low maintenance thanks to the absence of internal combustion mechanism and moving parts. The major disadvantage is that production, transportation and (on-site) storage of (hydrogen) fuel is costly. Like any other flammable fuel such as gasoline and natural gas, there are concerns about explosion of the hydrogen tank under specific conditions. FCs with liquid electrolyte (AFCs, MCFCs, PAFCs) might leak, and FCs with solid electrolyte (SOFCs) might crack. Note that PEMFCs contain solid and flexible electrolyte with no risk of leak or crack.

\section{F. Hydrogen storage}

Hydrogen, as one of the most abundant element on Earth, is nowadays considered as electricity storage medium. Unfortunately, the molecular hydrogen $\mathrm{H}_{2}$ is not readily available and must be first produced from e.g. hydrocarbon fuels (e.g. methane, coal and heavy residual oil), various biological materials or from the electrolysis of water. Excess green energy can be used to produce gaseous hydrogen and then to compress or liquefy it in order to store it in storage tanks. The stored hydrogen is converted back to electricity during peak periods using e.g. fuel cell for which hydrogen acts as a fuel. Reformation with steam of natural gas (often methane) or biogas (also called renewable natural gas, biomethane, swamp gas or landfill gas) is the most prevalent way to produce hydrogen. Hydrogen can also be produced by gasification of hydrocarbon fuels (often coal) or biomass in the presence/absence of oxygen. Electrolysis of water is insignificantly used to produce a small portion of overall hydrogen production today. It is often done using alkaline $(\mathrm{KOH})$ or proton exchange membrane (PEM) as electrolyte, and directly breaks down water into hydrogen and oxygen $\left(\mathrm{H}_{2} \mathrm{O}+e^{-} \rightarrow \mathrm{H}_{2}+\frac{1}{2} \mathrm{O}_{2}\right)$. A very detailed review of critical challenges, major R\&D needs and key benefits of all hydrogen-based storage technologies is given [16].

\section{G. Thermal energy storage (TES)}

A TES system can store off-peak excess electricity in the form of thermal energy, and releases the stored heat/cool during peak periods in order to be directly used for heating/cooling purposes or to be converted into electricity. One example is freezing water into ice (known as ice storage) in non-corrodible tanks and use the cool of the stored ice for air conditioning at a later point in time. Another newer form of TES is the molten salt technology where a mixture of molten salts (e.g. sodium nitrate and potassium nitrate) filled in the low-temperature tank (about e.g. $260 \mathrm{C}^{\circ}$ ) is pumped through evacuated tubes to be heated up by solar collectors up to e.g. $550 \mathrm{C}^{\circ}$. The hot molten salt then flows to a well-insulated high-temperature tank that can efficiently retain/store thermal energy for up to a week. When electricity is needed during peak periods, the stored hot molten salt flows into a heat exchanger into which feed water is also simultaneously piped from a water storage tank. The hot molten salt heats the water creating superheated steam. The cooled molten salt returns to the low-temperature tank, and the produced steam flows into a conventional steam turbine generating electricity. From the turbine the steam condenses back into water returning to the water storage tank.

\section{H. (Super)capacitor}

A conventional capacitor is an electrical device that basically consists of two electrical metal plates (conductors) e.g. aluminum, separated by a thin (often solid) insulating layer (dielectric) e.g. glass, mica, paper, air, plastic, ceramic. There are also electrolyte-based capacitors that employ moist dielectric. During charging process, electricity is stored in a static electric field that is being developed across the dielectric. The amount of electrical energy that a capacitor can store in its electric field is proportional to its capacitance and the square of the charging voltage $\left(E=\frac{1}{2} C V^{2}\right)$. When needed, the capacitor can be discharged releasing the stored electric energy very much like a rechargeable battery. Capacitance in general depends on geometry of the conductors (surface area as well as distance between conductors) and the dielectric properties (relative permittivity). These factors, thus, create fundamental practical limits for manufacturing highcapacitance standard capacitors. Moreover, the dielectric has a certain electric field strength resulting in a breakdown voltage limit. Supercapacitors (also known as ultra or double-layer or electro-chemical capacitors) are special class of capacitors that do not have a conventional dielectric. They, instead, utilize two metal plates coated with a porous carbon-based materials soaked in an (aqueous or organic) electrolyte, that are separated by a vanishingly thin insulator. The super capacitors can achieve high capacitance (and thus much larger energy density compared to standard capacitors) (up to $12 \mathrm{kF}$ ) thanks to the much bigger surface area of porous carbon-based plates to more effectively store the charges, and extremely small separation distance between them. The key disadvantage of supercapacitors is their low voltage limit (up to $5 \mathrm{~V}$ ), lower specific energy, higher self-discharge and their higher price compared to e.g. lithium-ion batteries. However they have much longer cycle life (theoretically any/unlimited number of times), shorter charging times, high specific power, and are more resilient to temperature variation (functioning well in hot/cold operating conditions). Moreover, unlike batteries, they do not require full-charge detection as, according to the circuit laws, they cannot be overcharged. A detailed overview of different classes of supercapacitors and their comparison is given in [17].

\section{ApPliCABILITY OF STORAGE TO THE BELGIAN GRID}

In this section, the applicability of the above-mentioned storage technologies to the Belgian power system will be discussed. Obviously, not all mentioned storage devices are equally applicable. Hydro storage requires sufficient water head and flow rate to be useful. Currently, in Belgium, only the PHES unit in Coo-Trois-Ponts is operational. This installation of $1100 \mathrm{MW}$ can provide five hours of power, totalling the stored energy to $5 \mathrm{GWh}$.

CAES systems could also be of interest to Belgium, because of the no longer operational coal mines in the east of the country. However, the utilisation of the caverns for air storage should be investigated. The process requires sufficiently large, stable and air-tight caverns to operate.

Flywheel storage and supercapacitors could be very interesting technologies as Belgium has a relative large amount of solar 


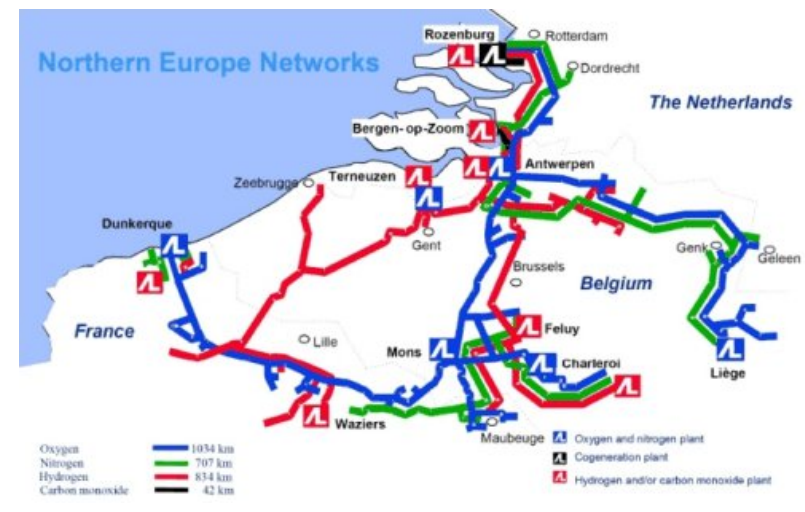

Fig. 4. Hydrogen pipe system in Belgium (Source: Air Liquide)

energy. As has been described in [18], trading solar energy on the stock market is not economically viable. The gains of selling the energy at the higher stock prices than those offered by fixed contracts are offset by the added risk due to the unpredictability of solar energy. Adding very dynamic storage units could manage the short-term variability and hence provide better economic revenue for solar plant owners. The same could be applicable to small wind turbine clusters, as the spatial smoothing of small clusters (2-6 turbines) is not high enough. However, the generally larger power of wind turbine clusters compared to solar panels increases the required power of the storage systems, increasing also the investment cost. Supercapacitor and flywheel storage are technologies with relative small energy content, but can effectively track RES power fluctuations in real time as these technologies do not suffer from quick charge/discharge cycles. This mark these technologies as high potential for short term $(\leq(15-30) \mathrm{min}$. optimization.

Unlike flywheel and (super)capacitor, batteries have limited charge/discharge cycles. Although it should be noted that newer technologies are less vulnerable to cycling, undercharging, deep discharging and high current, due to the chemical processes involved, the dynamic behaviour of batteries is slower than the aforementioned technologies. This makes battery storage more suited to medium power and energy applications. In contrast to the former mentioned technologies, batteries are a mature technology and are readily available on the market. Since for stationary grid support and economic optimization the energy density is not a big concern (in contrast to automotive applications), cheaper technologies like leadacid are also feasible.

Also hydrogen and fuel cells have potential in Belgium. In 2012, a 1 MW fuel cell has been installed in the Port of Antwerp. It utilizes residual hydrogen from the Solvin plant. Although this plant is designed to produce base load, it can be the first step to incorporate hydrogen power in the electricity grid on a large scale. Belgium is also suited as a test location for hydrogen power plants, as there is an extended hydrogen pipe net available. This pipe grid is connected to the (sea) ports of Rotterdam (The Netherlands), Antwerp, Zeebrugge and Ghent (Belgium) and is also connected to industrial sites in Charleroi (Belgium) and Waziers (France), as shown in Fig. 4, totalling more than $800 \mathrm{~km}$. As a gas pipeline has the potential to be utilised as a storage buffer, this is a major benefit to be available. However, it should be noted that the major part of hydrogen today is produced by steam reforming of natural gas and as by-product in chemical processing.

\section{CONCLUSIONS}

Reliable and affordable energy storage has a pivotal role in today's power systems with ever-increasing penetration of RES. Although comparing one energy storage solution with another one seems to be pointless as there exists no single optimal solution that suits all applications. However, a technoeconomic assessment of different energy storage technologies can provide guidelines to find (the combination of) the best available solution(s) for a particular case. This paper provides a high-level overview of widely-used storage technologies, their benefits and shortcomings. The structure of the Belgian electricity market is also presented. Finally, the technical applicability and economical viability of different storage devices to the Belgian power network is discussed.

\section{REFERENCES}

[1] Z. Iverson, A. Achuthan, P. Marzocca, D. Aidun, "Optimal design of hybrid renewable energy systems (HRES) using hydrogen storage technology for data center applications," Renewable Energy, vol. 52, pp. 79-87, Apr. 2013.

[2] S. Gill, G. W. Ault, I. Kockar, "The optimal operation of energy storage in a wind power curtailment scheme," In proc. IEEE Power and Energy Society General Meeting, July 2012.

[3] Elia, The day-ahead hub: a platform at the centre of ARPs activities, May 2012.

[4] Elia, The intraday hub: energy exchanges between ARPs to deal with unforeseen circumstances, Apr. 2012.

[5] Elia, Day-Ahead Market Coupling ensuring better market liquidity, May 2012.

[6] Synergrid, "Synthetic load profiles (slp)," http://www.synergrid.be/index.cfm?PageID=16896, 032013.

[7] Elia, Imbalance tariffs: compensation tariffs as an incentive for ARPs to maintain balance in their balancing perimeter, Feb. 2012.

[8] S. Avril, G. Arnaud, A. Florentin, M. Vinard,“ Multi-objective optimization of batteries and hydrogen storage technologies for remote photovoltaic systems," Energy, vol. 35, Issue 12, pp. 5300-5308, Dec. 2010.

[9] E. Mashhour and S. Moghaddas-Tafreshi, "Bidding strategy of Virtual Power Plant for participating in energy and spinning reserve markets; part I: Problem formulation," IEEE Trans. Power Syst., vol. 26, no. 2, pp. 949-956, May 2011.

[10] C.-J. Yang, R. B. Jackson, "Opportunities and barriers to pumpedhydro energy storage in the United States," Renewable and Sustainable Energy Reviews, vol. 15, Issue 1, pp. 839-844, Jan. 2011.

[11] C. A. Frangopoulos, "Optimization Methods for Energy Systems" book chapter in Exergy, Energy System Analysis, and Optimization, Editor: Christos A. Frangopoulos, 2007.

[12] R. C. Bansal, "Optimization methods for electric power systems: An overview," International Journal of Emerging Electric Power Systems, vol. 2, Issue 1, pp. 1-25, 2005.

[13] R. Baños, F. Manzano-Agugliaro, F.G. Montoya, C. Gil, A. Alcayde, J. Gómez, "Optimization methods applied to renewable and sustainable energy: A review," Renewable and Sustainable Energy Reviews, vol. 15, Issue 4, pp. 1753-1766, May 2011.

[14] K. C. Divya, J. Østergaard, "Battery energy storage technology for power systems-An overview," Electric Power Systems Research, vol. 79, Issue 4, pp. 511-520, Apr. 2009.

[15] N.-K. C. Nair, N. Garimella, "Battery energy storage systems: Assessment for small-scale renewable energy integration," Energy and Buildings, vol. 42, Issue 11, pp. 2124-2130, Nov. 2010.

[16] T. Lipman, "An Overview of Hydrogen Production and Storage Systems with Renewable Hydrogen Case Studies," May 2011.

[17] M. S. Halper, J. C. Ellenbogen, "Supercapacitors: A Brief Overview," March 2006.

[18] B. Zwaenepoel, J. Laveyne, L. Vandevelde, T. Vandoorn, B. Meersman, and G. Van Eetvelde, "Solar commercial virtual power plant," In proc. IEEE Power and Energy Society General Meeting, July 2013. 